1.59), 2.03 (1.007-4.13), respectively]. Males who slept $\geq 10$ hours/night had a $40 \%$ increase in cancer incidence and 59\% increase in cancer-caused mortality than males who slept 7 8 hours/night $[\mathrm{HR} \quad(95 \% \mathrm{CI})=1.40(1.04-1.88)$ and 1.59 (1.01-2.49), respectively]. There was an interaction effect between night-shift work of $\geq 20$ years and sleep of $\geq 10$ hours/night on cancer incidence $\left(P_{\text {interaction }}=0.027\right)$.

Conclusion For male subjects, both long night-shift work $(\geq 20$ years) and nighttime sleep duration ( $\geq 10$ hours) were independently and jointly associated with higher cancer incidence.

\section{SHIFTWORK AND BREAST CANCER: EPIDEMIOLOGY, BURDEN, AND IMPLICATIONS FOR PREVENTION}

\footnotetext{
${ }^{1}$ Manisha Pahwa*, 2,3 France Labrèche, $1,4,5 \mathrm{M}$ Anne Harris, ${ }^{1,6}$ Joanne Kim, ${ }^{1}$ Chaojie Song, 1,5,7 Paul A Demers. 'Occupational Cancer Research Centre, Cancer Care Ontario, Toronto, Canada; 'Institut de recherche Robert-Sauvé en santé et en Sécurité du travail, Montréal, Canada; ${ }^{3}$ School of Public Health, Université de Montréal, Montréal, Canada; ${ }^{4}$ School of Occupational and Public Health, Ryerson University, Toronto, Canada; ${ }^{5}$ Dalla Lana School of Public Health, University of Toronto, Toronto, Canada; ${ }^{6}$ Department of Epidemiology, Biostatistics and Occupational Health, McGill University, Montréal, Canada; ${ }^{7}$ CAREX Canada, Simon Fraser University, Burnaby, Canada
}

\subsection{6/oemed-2018-ICOHabstracts. 1395}

Introduction Approximately one in five workers globally work night, evening, or rotating shifts. Shiftwork involving circadian disruption is a probable carcinogen for breast cancer. Our objective was to synthesise the current state of the epidemiological literature, report on shiftwork-associated breast cancer burden in Canada, and discuss implications for prevention.

Methods A search was conducted for meta-analyses accompanied by a systematic review, published from 2010-2017, that included at least one meta-risk estimate (mRE) for breast cancer associated with any permanent/rotating night work exposure metric. For each included meta-analysis, heterogeneity values were extracted and an eight-point checklist was used to evaluate quality. An attributable fraction (AF) range for breast cancer, based on mREs from high quality meta-analyses and Canadian shiftwork survey data, was calculated using Levin's equation.

Results Seven meta-analyses collectively included 30 cohort and case-control studies spanning 1996-2016. Most reported statistically significant heterogeneity. In 5 meta-analyses that scored $\geq 6$ points on the quality assessment checklist, mREs for ever/never night shiftwork exposure ranged from 1.15 (95\% confidence interval $[\mathrm{CI}]: 1.05$ to $1.25, \mathrm{n}=9$ studies) to 1.40 (95\% CI: 1.13 to $1.73, \mathrm{n}=9$ studies). Using these mREs as lower and upper values in Levin's equation, the AF for breast cancer among the 1.5 million Canadian women who ever worked night/rotating shifts during 1961-2001 ranged from $2.04 \%-5.23 \%$. This corresponds to an estimated $460-$ 1180 annual incident breast cancers probably due to shiftwork; nearly half (200-510) are diagnosed among women in health care and social assistance.

Discussion Summaries of 20 years of epidemiological evidence support shiftwork as a probable breast carcinogen, but considerable heterogeneity between studies poses a challenge for precisely evaluating breast cancer risk and burden. Given the potentially substantial burden of breast cancer due to shiftwork, applied research on workplace-based prevention of circadian disruption is acutely needed to identify effective solutions for sectors where shiftwork prevalence is high.
977 A COMPARATIVE STUDY OF HEALTH STATUS OF SHIFT WORKERS AND NON-SHIFT WORKERS IN AN AUTOMOBILE INDUSTRY

Shriniket Mishra*. Hero MotCorp Limited, New Delhi, India

10.1136/oemed-2018-ICOHabstracts. 1396

Introduction In today's industrialised world, focus of mass producing organisations is to achieve maximum output and optimum utilisation of human resource by deploying workers in shift duties. This helps industries in achieving production targets but it adversely affects health of workers which is a well-established fact. Shift duties cause changes in circadian rhythm of human body which leads to adverse effects on physical and psychological health. This study was conducted at a large automobile organisation for comparing health status of shift workers with non-shift workers, establish cause and make improvement in health of shift workers.

Methods A cross sectional study was done through a questionnaire based survey and medical examination of workers. Two groups of 200 workers each was selected, one group was involved in shift work for 8 years and other group was working in day shift only for same number of years. A detailed questionnaire containing personal, occupational and medical history was presented to both groups. All data related to findings of medical examinations and survey was analysed to reach a conclusion.

Results There were significant differences in health status of both group of workers.76\% shift workers were suffering from G.I disorders- Indigestion, constipation, hyperacidity etc. compared to $18 \%$ of non-shift workers, $22 \%$ shift worker had Diabetes compared to $9 \%$ in non-shift workers, $36 \%$ shift workers had hypertension compared to $14 \%$ in non-shift, $44 \%$ shift workers had sleep disorder compared to $19 \%$ and $42 \%$ shift workers were obese compared to $26 \%$ of non-shift workers.

Discussion Results of study show that there are less health issues in non-shift workers compared to shift workers. This provides us evidence that if shift work can't be avoided then organisations should have scientific plans to minimise health hazards of shift work and more focus should be given to health of shift workers.

\section{Small Scale Enterprises and Informal Sector}

\section{SMALL + SAFE + WELL: UNDERSTANDING THE RELATIONSHIP BETWEEN PROGRAMS, ORGANISATIONAL CLIMATE, AND OUTCOMES FOR HEALTH, SAFETY AND WELLBEING AMONG SMALL BUSINESS}

Natalie Schwatka*, Liliana Tenney, Erin Shore, Josh Scott, Miranda Dally, Carol Brown, Lee Newman. University of Colorado Denver, Colorado School of Public Health, Centre for Health, Work, and Environment, Aurora, USA

\subsection{6/oemed-2018-ICOHabstracts. 1397}

Introduction The majority of workers are employed by small enterprises; however, small enterprises face many barriers to building and maintaining cultures of health and safety. This presentation will describe preliminary data from the evaluation 
of an existing community-based Total Worker Health (TWH) intervention - Health Links plus senior-level TWH leadership training - which aims to build small business capacity around health, safety and wellbeing policies and programs as well as strengthen organisational climates.

Methods We are recruiting small businesses with fewer than 500 employees in a variety of high and low hazard industries in Colorado USA. Upon enrolment, businesses are randomised into one of two doses:

- Health Links plus senior-level TWH leadership training and

- Health Links.

Annual business- and employee-level assessments are collected.

Results Data collection began in April 2017 and we will have baseline data to share from about 100 businesses and 3500 employees. We will present a cross-sectional assessment of (1) organizational-level adoption of TWH policies and programs (2) organisational safety and health climates, and (3) workerlevel health and safety outcomes.

Preliminary data from businesses enrolled in Health Links prior to the start of the study $(n=145)$ indicates variation in level of policies and programs by business. Thus, we hypothesise that small businesses that have more TWH policies and programs have (1) more positive organisational safety and health climates, and (2) safer and healthier workers.

Discussion Little is known about small business capacity for delivering health, safety and wellbeing. Even less is known about how TWH interventions impact employee safety, health and well-being in small enterprises. This information is needed to understand how to creategeneralizable TWH interventions that can have a broad public health impact.

\section{VALUE ON INVESTMENT FOR SMALL BUSINESS SAFETY, HEALTH AND WELLBEING}

${ }^{1}$ Natalie Schwatka*, 'Lee Newman, ${ }^{2}$ Claire Brockbank, 'Liliana Tenney, ${ }^{1}$ Miranda Dally, ${ }^{3}$ Kim Jinnett, ${ }^{4}$ Adam Atherly, ${ }^{5}$ Ron Goetzel, ${ }^{6}$ James McMillen, 'David Weitzenkamp, ${ }^{1}$ Derek Smith, 'Erin Shore. ${ }^{1}$ University of Colorado Denver, Colorado School of Public Health, Centre for Health, Work, and Environment, Aurora, USA; ${ }^{2}$ Segue Consulting, Denver, USA; ${ }^{3}$ Integrated Benefits Institute and Centre for Workforce Health and Performance, San Francisco, USA; ${ }^{4}$ University of Colorado Denver, Colorado School of Public Health, Department of Health Systems, Management, and Policy, Aurora, USA; ${ }^{5}$ Johns Hopkins University Bloomberg School of Public Health, Institute for Health and Productivity Studies, and IBM Watson Health, Bethesda, USA; ${ }^{6}$ Pinnacol Assurance, Denver, USA

10.1136/oemed-2018-ICOHabstracts. 1398

Introduction Health promotion programs may succeed in midto large-size companies, but what can be done to reach the small enterprises where the majority of people work? Furthermore, how successful can these efforts be and what is the value of investing in small business employee health? This presentation will examine the nexus of workers' compensation (WC), health promotion, productivity and small businesses.

Methods Three-hundred and fourteen businesses and 16926 of their employees participated in a multi-year worksite wellness program offered by a WC insurer in the United States from 2010-2014. Yearly self-report health risk assessment data and WC claims data were collected. In a series of studies using logistic and generalised linear models, we examined the relationship between employee health, safety and productivity. Additionally, using data from employees who participated in the program for $2+$ years $(n=5,766)$, we examined the change in health during the WWP.

Results A greater number of small businesses ( $<500$ employees) participated in the WWP than large businesses (500 +employees). Additionally, small businesses had higher employee participation rates than large businesses.

WC claims were best predicted by the occurrence of a previous WC claim and poor behavioural health (e.g., depression). Productivity at work was best predicted by previous WC claim, increasing numbers of chronic health conditions, and work task difficulty.

Small businesses gained the most from the WC insurerdriven WWP, compared to large businesses. Small businesses (<500 employees) saw improvements in job health culture, stress, depression, overall health rating, smoking, nutrition, and exercise from baseline to 1 st and 2nd follow-up.

Discussion Our results demonstrate the value of investing in employee health generally and the value of doing so through local, intermediary organisations. Employers who control jobsite safety hazards and promote employee health can observe gains in productivity and reductions in injury.

\section{HEALTH INEQUALITIES, WORK AND ACCESS TO SOCIAL SECURITY OF INFORMAL WORKERS IN LATIN AMERICA, AFRICA AND ASIA}

O Solar*, P Bernales, MJ Gonzalez, A Riesco, A Vives, V Alamos. Work, Employment and Health Inequities Faculty of Social Science of Latinamerica (FLACSO), Santiago Chile

\subsection{6/oemed-2018-ICOHabstracts.1399}

Introduction Around the world exist the expansion of the informal economy and traditionally people -working in the informal economy (WIE) do not have access to the established state-run healthcare systems. Understanding the barriers that explain the low participation of people-working informal economy in health systems and social security systems, providing social protection and eliminate obstacles to accessing care, should be viewed by governments as a strategy to improve the health of these workers.

Methods Develop baseline of information on the health and occupational health of informal workers and their access to health and social security systems in 6 countries of Africa, 6 of Asia and 12 countries of Latin America. The methodology included Case Studies were oriented to analyse access to health and social security, their employment and working conditions and their relationship to the characteristics of the countries' economic structure. The Qualitative Studies Component collected the primary information based on the informal workers' perception The Core Indicators component aimed to build indicators on several domains and subdomains of informal work and health that are relevant, robust and comparable between countries, as well as to identify information gaps, based on the analysis of different country surveys.

Result People -WIE are women and men from different age groups - higher rate of work among eldest and youngest groups - and educational backgrounds levels, living in urban and rural areas; present in different industrial sectors -; strong 